\title{
Pancreas
}

\section{PTU-030 DO OLEIC ACID AND N-3 FATTY ACIDS PREVENT PANCREATIC CANCER? DATA FROM A UK PROSPECTIVE STUDY USING 7-DAY FOOD DIARIES}

doi:10.1136/gut.2011.239301.158

P J R Banim, ${ }^{1 *}$ R Luben, ${ }^{2}$ A McTaggart, ${ }^{2}$ K-T Khaw, ${ }^{2}$ N Wareham, ${ }^{3}$ A R Hart ${ }^{1,4}{ }^{1}$ School of Medicine, University of East Anglia, Norwich, UK; ${ }^{2}$ Institute of Public Health, Cambridge, UK; ${ }^{3}$ MRC Epidemiology, University of Cambridge, Cambridge, UK; ${ }^{4}$ Gastroenterology, Norfolk and Norwich University NHS Trust, Norwich, UK

Introduction There are plausible biological mechanisms for how both oleic acid and n-3 fatty acids may prevent the development of pancreatic cancer through their effects on cancer oncogenes, apoptosis and inflammatory processes. The aim of this study was to investigate the effect of these nutrients in a prospective cohort study, for the first time using sevenday food diaries (7-DFDs), the most accurate feasible method of estimating food intake in large scale epidemiological investigations.

Methods 25639 participants (56\% women), aged 40-74 years were recruited into the European Prospective Investigation into Cancer-Norfolk (EPIC-Norfolk) with 23658 completing 7-DFDs at enrolment. The cohort was monitored for 16 years, using hospital records and cancer registry data to identify participants who developed pancreatic cancer with all notes reviewed to confirm diagnosis. The 7-DFDs recorded one week's diet including food types, frequency of consumption, quantities and cooking methods. A representative sample of 3970 as well as those with gallstone disease had their 7-DFDs coded by nutritionists using a computer program containing nutrient information on 11000 food and 55000 portion sizes. A cohort analysis used Cox regression to estimate sex specific hazard ratios (HR) for developing pancreatic cancer comparing fifths of: total dietary n-3 fatty acids, eicosapentaenoic acid (EPA), docosapentaenoic acid (DHA) and oleic acid intake, adjusted for the risk factors of age, gender, total energy intake, smoking and diabetes.

Results A total of 88 participants with a mean age at diagnosis of 72.2 years ( $S D=9.0$ years) $(55.5 \%$ women) developed pancreatic cancer with $77 \%$ having advanced disease. The interval between recruitment and diagnosis was 7.9 years $(\mathrm{SD}=3.7$ years). Increased oleic acid intake was associated with a decreased risk of developing pancreatic cancer (highest fifth (23.7-78.3 g/day) versus lowest fifth $(2.5-13.2 \mathrm{~g} /$ day), $\mathrm{HR}=0.2995 \%$ CI 0.09 to 0.91 ) with a significant trend across fifths (per fifth increase, HR=0.72 95\% CI 0.55 to 0.96 $p=0.023)$. The population attributable fraction for an intake of less than $23.7 \mathrm{~g} /$ day of oleic acid was $32.1 \%$. No associations were found for total dietary n- 3 fatty acids (highest vs lowest fifth, $\mathrm{HR}=0.5595 \%$ CI 0.24 to 1.30 ) or dietary EPA and DHA intake.

Conclusion An increased oleic acid intake may be involved in the aetiology of one-third of pancreatic cancer cases. No associations were found for n3 PUFA's. The findings suggest oleic acid should be measured in future aetiological work of this aggressive cancer.

Competing interests None.

Keywords Aetiology, Fatty acids, Pancreatic cancer. 